\title{
$\mathrm{TiCuO}$ 薄膜的显微结构对 $\mathrm{Cu}$ 离子 释放和内皮细胞行为的影响
}

\author{
程 丹 ${ }^{1}$, 黄 斌 ${ }^{1}$, 陈 涛 $^{1}$, 景凤娟 ${ }^{1}$, 谢 东 $^{2}$, 冷永祥 ${ }^{1}$, 黄 楠 $^{1}$
}

(西南交通大学 1. 材料科学与工程学院, 先进材料技术教育部重点实验室; 2. 物理科学与技术学院, 成都 610031)

摘 要: 采用直流磁控溅射技术, 在 $\mathrm{Si}$ 片和 $316 \mathrm{~L} \mathrm{SS}$ 基体上制备了不同 $\mathrm{Cu}$ 含量的 $\mathrm{TiCuO}$ 薄膜。采用 $\mathrm{X}$ 射线衍射仪 $(X R D)$ 、透射电镜 $(T E M) 、 X$ 射线能谱仪(EDS)和 $X$ 射线光电子能谱 $(X P S)$ 对薄膜的显微结构和化学组成进行了分 析。采用电化学腐蚀和模拟体液浸泡实验评价了薄膜的腐蚀性能和 $\mathrm{Cu}$ 离子释放特性。体外静态培养内皮细胞后, 采 用细胞计数试剂盒(CCK-8)评价了 $\mathrm{TiCuO}$ 薄膜的细胞活性。研究结果表明, 未掺杂的 $\mathrm{TiO}_{2}$ 薄膜为金红石相, 掺入 $\mathrm{Cu}$ 后的 $\mathrm{TiCuO}$ 薄膜由非晶基体上含有 $\mathrm{Cu}_{2} \mathrm{O}$ 的纳米晶粒构成。薄膜中的富 $\mathrm{Cu}$ 区引起了薄膜腐蚀。含 $\mathrm{Cu}$ 量高的 $\mathrm{TiCuO}$ 薄膜更易被腐蚀, 并释放出较多 $\mathrm{Cu}$ 离子。 $\mathrm{TiCuO}$ 薄膜释放出一定浓度的 $\mathrm{Cu}$ 离子促进了内皮细胞活性。研 究表明, $\mathrm{TiCuO}$ 薄膜的含 $\mathrm{Cu}$ 量和显微结构影响了 $\mathrm{Cu}$ 离子释放, 对其内皮细胞活性起了关键作用。

关 键 词: $\mathrm{TiCuO}$ 薄膜; 磁控溅射; $\mathrm{Cu}$ 离子释放; 内皮细胞治性

中图分类号: TG178; R318 文献标识码: A

\section{Microstructure of TiCuO Films on Copper Ion Release and Endothelial Cell Behavior}

\author{
CHENG Dan ${ }^{1}$, HUANG Bin ${ }^{1}$, CHEN Tao ${ }^{1}$, JING Feng-Juan ${ }^{1}$, XIE Dong ${ }^{2}$, LENG Yong-Xiang ${ }^{1}$, HUANG Nan ${ }^{1}$
}

(1. Key Laboratory for Advanced Technologies of Materials, Ministry of Education, School of Materials Science and Engineering, Southwest Jiaotong University, Chengdu 610031, China; 2. School of Physics Science and Technology, Southwest Jiaotong University, Chengdu 610031, China)

\begin{abstract}
TiCuO}$ films with different microstructures and $\mathrm{Cu}$ contents were prepared on $\mathrm{Si}$ and 316L SS substrates using DC magnetron sputtering. The microstructure and chemical composition of the films were analyzed by X-ray diffraction (XRD), transmission electron microscopy (TEM), X-ray energy dispersive spectroscopy (EDS), and X-ray photoelectron spectroscopy (XPS). Corrosion behavior and $\mathrm{Cu}$ ions release properties of the films were evaluated by electrochemical corrosion and simulated body-fluid immersion tests, respectively. Endothelial cell (EC) viability on the films was evaluated by CCK-8 assay in vitro. Un-doped $\mathrm{TiO}_{2}$ films exhibit rutile phases. After $\mathrm{Cu}$ incorporation, the $\mathrm{TiCuO}$ films consist of nano-crystalline grains of $\mathrm{Cu}_{2} \mathrm{O}$ on their amorphous matrix. The films with higher $\mathrm{Cu}$ contents are more susceptible to corrosion and release more $\mathrm{Cu}$ ions. The $\mathrm{Cu}$-rich phases of the films result in corrosion. $\mathrm{EC}$ viability is enhanced by a certain concentration of $\mathrm{Cu}$ ions released from $\mathrm{TiCuO}$ films. All above results demonstrated that microstructures and $\mathrm{Cu}$ contents of $\mathrm{TiCuO}$ films play an important role in controlling copper ions release behaviors, and therefore affect the EC viability.
\end{abstract}

Key words: $\mathrm{TiCuO}$ films; magnetron sputtering; $\mathrm{Cu}$ ions release; ECs viability 
目前心脑血管疾病的发病率逐年升高, 严重威胁 人类的健康。人工血管支架、人工心瓣、人工血泵等 植入性医疗器械是治疗心脑血管疾病的有效手段。 然而人体在使用这些植入物后会出现的血栓、内膜 增生等问题已成了急需解决的重要问题。其原因是 心血管器械在植入时会对血管内皮造成损伤, 并且 由于制备这些器械的材料, 如 316 不锈钢、钴合金、 钛及钛合金等材料的表面不具有生物活性, 可能会 对血管造成内膜损伤而难以及时修复。同时所造成 病理状态的血管内皮细胞会促进炎症、血栓和平滑 肌细胞增殖等不良反应 ${ }^{[1]}$ 。正常功能的内皮细胞是 防止血栓形成的天然屏障, 所以在生物材料表面上 完全快速内皮化, 可有效抑制血栓和平滑肌增殖等 不良反应 ${ }^{[2]}$ 。

由于钛及钛合金具备优良的机械性能、延展性和 生物相容性, 临床上广泛用作医疗器械。Akahori 等 ${ }^{[3]}$ 发现钛及钛合金优良的血液相容性是由于其表面生 成的稳定氧化物二氧化钛的作用。黄楠等 ${ }^{[4-5]}$ 研制得 到的氧化钛薄膜具有较好的生物相容性, 可以提高 316L SS 支架的耐腐蚀性、化学惰性和血液相容性。 但氧化钛薄膜还未达到理想的抗凝血要求, 服役期 间病人还需服用抗凝药。

金属 $\mathrm{Cu}$ 离子是人体必需的微量元素, 正常人 体中含有约 $100 \sim 120 \mathrm{mg}$ 的 $\mathrm{Cu}$ 元素 ${ }^{[6]}$ 。人体服用 $\mathrm{Cu}$ 的药用浓度高于 $5.0 \mathrm{mg} / \mathrm{kg}$ 体重时才表现出毒性 ${ }^{[7]}$, $\mathrm{Cu}$ 还是人体生命过程中许多重要酶的活性中心 ${ }^{[8]}$ 。 研究发现, 一定浓度的 $\mathrm{Cu}$ 离子可以促进内皮细胞 的增殖, 但并不促进平滑肌和成纤维细胞的增 殖 $^{[9-10]}$, 还可以通过上调血管内皮生长因子的表达 来促进血管生成和血管成熟 ${ }^{[11]}$, 因此, 合金材料中 掺入适量的 $\mathrm{Cu}$, 不仅不会出现毒性, 反而会起到促 进内皮细胞增殖的作用。但在合金中掺入多少 $\mathrm{Cu}$ 、 如何掺 $\mathrm{Cu}$ 才能使合金保持其优良的生物相容性并 促进血管内皮活性, 需要进一步研究。

本研究在氧化钛体系中加入 $\mathrm{Cu}$ 元素, 利用磁 控溅射技术制备出不同含 $\mathrm{Cu}$ 量的 $\mathrm{TiCuO}$ 薄膜, 以 释放 $\mathrm{Cu}$ 离子和促进内皮细胞活性。目前, $\mathrm{TiCuO}$ 薄 膜的研究主要集中在其光催化 ${ }^{[12]}$ 和抗菌性能 ${ }^{[13-14]}$ 等, 很少有研究关注其内皮细胞相容性, 也较少有 人采用透射电镜研究 $\mathrm{TiCuO}$ 薄膜的显微结构。本研 究主要研究了 $\mathrm{TiCuO}$ 薄膜的组成、结构、电化学腐 蚀、 $\mathrm{Cu}$ 离子释放和内皮细胞行为, 并分析了薄膜的 成分和显微结构对腐蚀、 $\mathrm{Cu}$ 离子释放和内皮细胞行 为的影响。

\section{1 实验方法}

\section{1 薄膜样品的制备}

采用直流磁控溅射(DCMS)技术, 改变溅射靶 材中的 $\mathrm{Cu}$ 含量, 在 $\mathrm{Si}$ 片和 $316 \mathrm{~L} \mathrm{SS}$ 基体表面制备 出不同 $\mathrm{Cu}$ 含量的 $\mathrm{TiCuO}$ 复合薄膜, 薄膜厚度约为 $200 \mathrm{~nm}$ 。在矩形钛靶(纯度 $99.99 \%$, 尺寸 $135 \mathrm{~mm} \times$ $170 \mathrm{~mm} \times 6 \mathrm{~mm}$ )的有效溅射区(跑道环)中嵌入各种 数量的铜柱(纯度 $99.97 \%$ ), 通过改变纯 $\mathrm{Ti}$ 靶中 $\mathrm{Cu}$ 条的镶嵌量, 得到 $\mathrm{Cu} /(\mathrm{Ti}+\mathrm{Cu})$ 面积比分别为 $2.15 \%$ 和 $14.5 \%$ 的 $\mathrm{Ti}-\mathrm{Cu}$ 镶嵌靶材。样品 $\mathrm{TiCuO}-1 、 \mathrm{TiCuO}-2$ 薄膜分别采用 $\mathrm{Cu} /(\mathrm{Ti}+\mathrm{Cu})$ 的面积比为 $2.15 \% 、 14.5 \%$ 的 $\mathrm{Ti}-\mathrm{Cu}$ 镶嵌靶材, 样品 $\mathrm{TiO}_{2}$ 薄膜采用钛靶(纯度 99.99\%)。设置真空室基础真空为 $2.0 \times 10^{-3} \mathrm{~Pa}$, 溅 射沉积气压为 $0.63 \mathrm{~Pa}, \mathrm{O}_{2} / \mathrm{Ar}$ 流量比为 $12 / 60$, 基片 偏压为 $-50 \mathrm{~V}$, 溅射靶材和样品的距离约为 $90 \mathrm{~mm}$, 溅射的直流电流为 $2 \mathrm{~A}$ 。

\section{2 材料表征方法}

采用日本 JSM-7001F 型扫描电子显微镜附件 X 射线能谱仪(EDS)检测 $\mathrm{Si}$ 片基体上沉积的 $\mathrm{TiCuO}$ 薄 膜的表面成分。使用表面台阶仪(美国 XP-2 型)测定 $\mathrm{Si}$ 片基体上 $\mathrm{TiCuO}$ 薄膜的厚度。采用 $\mathrm{X}$ 射线光电 子能谱仪(SPECS-XPS，德国)分析 $\mathrm{Si}$ 片基体上 $\mathrm{TiCuO}$ 薄膜表面的化学键合状态, XPS 设备阳极靶 为铝靶, 测试功率为 $12 \mathrm{kV} \times 315 \mathrm{~mA}$, 真空度为 $2 \times 10^{-7} \mathrm{~Pa}$ 。采用 $\mathrm{X}$ 射线衍射仪(Philips $\chi$ Pert, 荷兰) 分析 $\mathrm{Si}$ 片基体上 $\mathrm{TiCuO}$ 薄膜的表面结构, 其中采 用 $\mathrm{CuK} \alpha$ 辐射 $(\lambda=0.15406 \mathrm{~nm}), \mathrm{X}$ 射线源管电压 $30 \mathrm{kV}$ 、 电流 $30 \mathrm{~mA}$ 、掠射角 $0.5^{\circ}, 2 \theta$ 扫描范围 $20^{\circ} \sim 70^{\circ}$ 。采用 透射电子显微镜(JEM-2100F JEOL, 日本)对 $\mathrm{TiCuO}$ 薄膜进行选区电子衍射和高分辨观察, 透射电镜的 样品制备流程包括: 采用 1.1 所述的工艺参数, 在 $\mathrm{NaCl}$ 晶体上沉积 $80 \mathrm{~nm}$ 的 $\mathrm{TiCuO}$ 薄膜, 用酒精和蒸 馏水(2: 1)混合液浸泡薄膜样品, 使 $\mathrm{NaCl}$ 基体和薄 膜分离, 用铌子取出薄膜碎片并干燥后, 将薄膜置 于铜网上进行 TEM 检测。采用电化学工作站(IM6, Zahner, 德国)测试在 $316 \mathrm{~L} \mathrm{SS}$ 上沉积的 $\mathrm{TiCuO}$ 薄膜 的电化学腐蚀性能, 选取 $\mathrm{pH}=7.4$ 的磷酸缓冲盐 (PBS)标准溶液作为腐蚀溶液, 扫描速度 $2 \mathrm{mV} / \mathrm{s}$, 电 位扫描范围-1 $0.8 \mathrm{~V}$ 。参考《医疗器械生物学评价》 标准, 根据 ISO 10993-12:2002 和 ISO 10993-15:2000, 将在 $\mathrm{Si}$ 片表面沉积的表面积为 $3 \mathrm{~cm}^{2}$ 的 $\mathrm{TiCuO}$ 薄膜 浸泡于 $2 \mathrm{~mL}$ 的标准 PBS ( $\mathrm{pH}=7.4)$ 中, 放置于 $37^{\circ} \mathrm{C}$ 恒温箱中, 分别浸泡 $30 \mathrm{~min}$ 和 $1 、 3 、 5 、 15 、 30 \mathrm{~d}$ 后取出释放液, 采用 ICP-MS 电感耦合等离子发射 
光谱仪(Agilengt 725 型)设备检测 $\mathrm{TiCuO}$ 薄膜在不 同时间内释放的 $\mathrm{Cu}$ 离子浓度。提取于脐带静脉的 内皮细胞分别在 $\mathrm{Si}$ 片基体上沉积薄膜的样品表面 培养 1 和 $3 \mathrm{~d}$ 后, 利用细胞计数试剂盒(Cell Counting Kit-8, CCK-8)表征内皮细胞活性。步骤为: 首先在 样品表面种植密度为 $5 \times 10^{4}$ cells $/ \mathrm{cm}^{2}$ 的内皮细胞, 培养 1 和 $3 \mathrm{~d}$ 后丢弃旧培养基, 接着将每个样品用 PBS 洗涤两次。再将预先配置的含有 $10 \% \mathrm{CCK}-8$ 的 新鲜培养基以 $350 \mu \mathrm{L} /$ 孔加入, 放置于 $37^{\circ} \mathrm{C} 、 5 \% \mathrm{CO}_{2}$ 条件下孵育 $3 \mathrm{~h}$, 吸取 $200 \mu \mathrm{L} /$ 孔的反应液到 96 孔板 中, 此过程注意避免光照。利用酶标仪 ( $\mu$ Quant, Bio-tek instruments Inc)在 $450 \mathrm{~nm}$ 波长下测量吸光度值。

\section{2 结果与讨论}

\subsection{TiCuO 薄膜的成分、微观结构与结合状态}

表 1 为采用 $\mathrm{EDS}$ 检测的 $\mathrm{TiCuO}$ 薄膜样品的成 分分析结果, 样品 $\mathrm{TiCuO}-1$ 和 $\mathrm{TiCuO}-2$ 中 $\mathrm{Cu}$ 分别 为 $12.4 \mathrm{at} \%$ 和 $23.1 \mathrm{at} \%$ 。

由直流磁控溅射制备的未掺杂的 $\mathrm{TiO}_{2}$ 样品是 金红石相结构(图 1)。含 $\mathrm{Cu}$ 量分别为 $12.4 \mathrm{at} \%$ 和 $23.1 \mathrm{at} \%$ 的 $\mathrm{TiCuO}$ 薄膜均为明显的非晶态结构。这是 因为 $\mathrm{Cu}$ 的掺入阻碍了薄膜中 $\mathrm{TiO}_{2}$ 的结晶行为 ${ }^{[15]}$, 导致了明显的非晶相形成。

采用 $\mathrm{TEM}$ 对 $\mathrm{TiCuO}$ 薄膜的显微结构进行了进 一步分析(图 2)。采用 TEM 获得的 TiCuO-1 样品的 表面形貌(图 2(a)), 可见在灰色基体 $\mathrm{A}$ 中出现了大 小不等的黑色颗粒 B, 颗粒大小约为 70 150 nm。
EDS 结果显示: B 区域的 $\mathrm{Cu}$ 含量大于 A 区域(图 2(b)), 因此 $B$ 区域是 $\mathrm{Cu}$ 的富集区。区域 $\mathrm{A}$ 的选区电子衍 射图谱显示(图 2(c)), TiCuO-1 样品的 A 区域未发现 明显的衍射斑点或衍射同心圆环，具有明显的非晶 衍射特征的衍射花样，通过 A 区域高分辨透射电子 显微镜(HRTEM)结果可见其为明显的非晶结构(图 2(e)), 与 XRD 的结果一致。B 区域中有少量衍射斑 点存在(图 2(d))。通过测量得到衍射晶面间距分别 为 $d=0.300 、 0.174$ 和 $0.151 \mathrm{~nm}$, 推测 $\mathrm{B}$ 区域为 $\mathrm{Cu}_{2} \mathrm{O}$ 相, 对应晶面分别是 $\mathrm{Cu}_{2} \mathrm{O}(110) 、 \mathrm{Cu}_{2} \mathrm{O}(211)$ 和

表 1 采用 EDS 检测 TiCuO 薄膜中的 Ti、Cu、O 元素的相对原子含量

Table 1 Atomic concentration of TiCuO films evaluated by EDS

\begin{tabular}{cccc}
\hline Sample & $\mathrm{Cu} / \mathrm{at} \%$ & $\mathrm{Ti} / \mathrm{at} \%$ & $\mathrm{O} / \mathrm{at} \%$ \\
\hline $\mathrm{TiCuO}-1$ & 12.4 & 12.3 & 75.3 \\
$\mathrm{TiCuO}-2$ & 23.1 & 6.7 & 70.2 \\
\hline
\end{tabular}

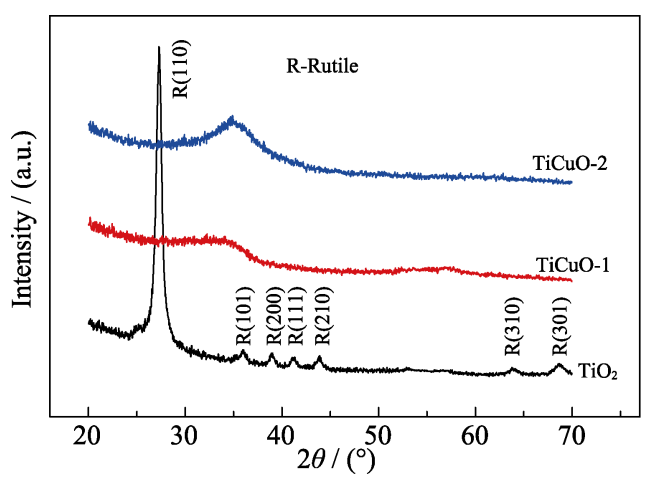

图 $1 \mathrm{TiO}_{2}$ 和 $\mathrm{TiCuO}$ 样品的 XRD 图谱

Fig. 1 XRD patterns of $\mathrm{TiO}_{2}$ and $\mathrm{TiCuO}$ samples
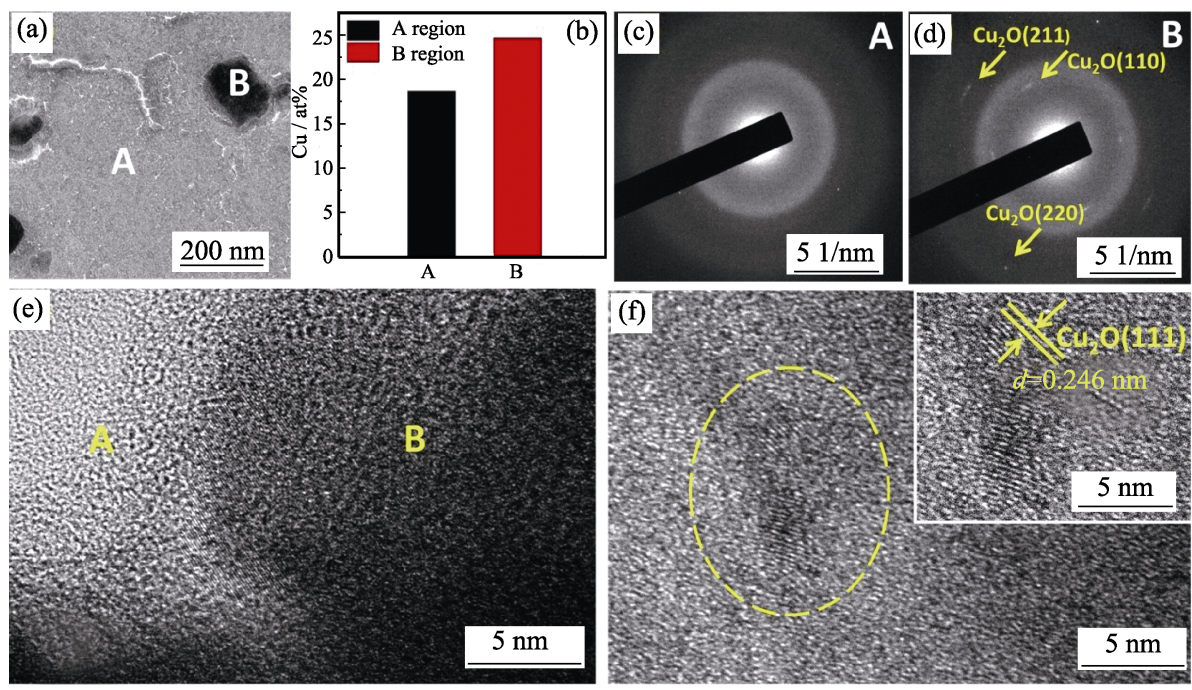

图 2 TiCuO-1 样品的 TEM 照片(a), A 和 B 区域 $\mathrm{Cu}$ 原子百分比(b), $\mathrm{A}(\mathrm{c})$ 和 B(d)区域的选区电子衍射图(c)及其 HRTEM 形貌(e) (f)

Fig. 2 TEM image (a), Cu contents evaluated by EDS (b), the selected area electron diffraction results of A region (c) and B region (d), and their corresponding HRTEM images (e, f), respectively, of the TiCuO-1 sample 
$\mathrm{Cu}_{2} \mathrm{O}(220)$ 。图 2(f)是 B 区域的 HRTEM 照片, 从图 中观察到大部分区域是非晶区(无条纹), 局部出现 了少量纳米晶(虚线框), 其晶粒大小约为 $10 \mathrm{~nm}$, 晶面间距为 $d=0.246 \mathrm{~nm}$, 因此推算衍射条纹可能 是 $\mathrm{Cu}_{2} \mathrm{O}(111)$ 晶面。结果显示, 含 $\mathrm{Cu}$ 量为 $12.4 \mathrm{at} \%$ 的 TiCuO-1 样品非晶基体上存在少量 $\mathrm{Cu}_{2} \mathrm{O}$ 纳米 晶粒。

TiCuO-2 样品的 TEM 表面形貌显示, 在薄膜灰 色基体 A 中也出现大小不等的黑色颗粒 B(图 3(a)), 其颗粒尺寸(约 70 300 nm)大于 TiCuO-1 样品中的 颗粒(约 70 150 nm)。EDS 线扫描结果(图 3(b))显 示, $\mathrm{B}$ 区域的含 $\mathrm{Cu}$ 量大于 $\mathrm{A}$ 区域, 因此 $\mathrm{B}$ 区域是 $\mathrm{Cu}$ 的富集区。图 3(c)是薄膜基体 $\mathrm{A}$ 的选区电子衍射, 可以看出有一个散漫的衍射环和一个较宽的亮环 ${ }^{[16-18]}$, 此较宽亮环的衍射晶面间距为 $0.246 \mathrm{~nm}$, 对应衍射 晶面为 $\mathrm{Cu}_{2} \mathrm{O}(111)$, 再通过图 3(e)中 $\mathrm{A}$ 区域的 HRTEM 照片可以看出区域局部存在晶粒, 测量得到晶面间 距 $d=0.213 \mathrm{~nm}$, 推测发生衍射的晶面是 $\mathrm{Cu}_{2} \mathrm{O}(200)$ 。 因此推测, 基体 $\mathrm{A}$ 区域内为非晶基体与少量细小的 $\mathrm{Cu}_{2} \mathrm{O}$ 纳米晶粒构成。图 3(d) 是颗粒 $\mathrm{B}$ 区域的选区电 子衍射图谱, 从中可明显看出有规则排列的衍射斑 点, 其晶面间距 $d$ 值分别为 $0.302 、 0.122$ 和 $0.128 \mathrm{~nm}$, 对应晶面为 $\mathrm{Cu}_{2} \mathrm{O}(110) 、 \mathrm{Cu}_{2} \mathrm{O}(222)$ 和 $\mathrm{Cu}_{2} \mathrm{O}(311)$ 。从 HRTEM 照片(图 3(f))中也可以看出 B 区域存在明显 的晶粒, 测得晶面间距是 $0.301 \mathrm{~nm}$, 对应衍射晶面 是 $\mathrm{Cu}_{2} \mathrm{O}(110)$ 晶面。因此, $\mathrm{TiCuO}-2$ 样品也为非晶结 构与 $\mathrm{Cu}_{2} \mathrm{O}$ 纳米晶粒共存。

图 4 是采用 $\mathrm{Ar}^{+}$离子刻蚀 $\mathrm{TiO}_{2}$ 和 $\mathrm{TiCuO}$ 样品
表面 $30 \mathrm{~nm}$ 后的 XPS 图谱, 表 2 是对 $\mathrm{TiO}_{2}$ 和 $\mathrm{TiCuO}$ 样品中的 $\mathrm{Ti} 2 \mathrm{p}$ 和 $\mathrm{Cu} 2 \mathrm{p}$ 进行拟合后, $\mathrm{Ti}^{4+} 、 \mathrm{Ti}^{3+} 、 \mathrm{Ti}^{2+}$ 在 Ti2p 图谱中所占面积的百分含量和 $\mathrm{Cu}^{2+} 、 \mathrm{Cu}^{+}$在 $\mathrm{Cu} 2 \mathrm{p}$ 图谱中的所占面积的百分含量。根据文献报道, $\mathrm{CuO}$ 的 $\mathrm{Cu} 2 \mathrm{p}$ 特征峰为 $\mathrm{Cu} 2 \mathrm{p}_{3 / 2}(933.5 \mathrm{eV})$ 和 $\mathrm{Cu} 2 \mathrm{p}_{1 / 2}$ $(953.5 \mathrm{eV}), \mathrm{Cu}_{2} \mathrm{O}$ 的 $\mathrm{Cu} 2 \mathrm{p}$ 峰在 $\mathrm{Cu} 2 \mathrm{p}_{3 / 2}(932.5 \mathrm{eV})$ 和 $\mathrm{Cu} 2 \mathrm{p}_{1 / 2}(952.5 \mathrm{eV})^{[19]}$, 其结合能比 $\mathrm{CuO}$ 的结合能低 $1 \mathrm{eV}$ 。图 4(a)的 $\mathrm{Cu} 2 \mathrm{p}$ 谱图显示, 含 $\mathrm{Cu}$ 量较多的 TiCuO-2 样品除了在 $933.5 \mathrm{eV}$ 处有 $\mathrm{CuO}$ 的 $\mathrm{Cu} 2 \mathrm{p}_{3 / 2}$ 特征峰以外, 在 940.5 948.5 eV 范围内还明显出现 了 $\mathrm{Cu}^{2+}$ 特征卫星峰, 这是 $\mathrm{CuO}$ 的典型特征峰 ${ }^{[20-22]}$ 。 图 4(a)和表 2 的结果均表明, TiCuO-1 和 TiCuO-2 样 品都含有 $\mathrm{Cu}_{2} \mathrm{O}$, 且含 $\mathrm{Cu}$ 量较多的 $\mathrm{TiCuO}-2$ 样品中 还含有较少的 $\mathrm{CuO}$ 。文献报道, 在 $\mathrm{Cu}$ 的氧化过程 中, 首先生成 $\mathrm{Cu}_{2} \mathrm{O}$ 结构, 然后 $\mathrm{Cu}_{2} \mathrm{O}$ 进一步被氧化 生成 $\mathrm{CuO}$ 结构 ${ }^{[23-24]}$, 同时杂质元素会影响 $\mathrm{Cu}$ 的氧 化过程 ${ }^{[25]}$ 。本研究认为, $\mathrm{TiCuO}$ 薄膜中的 $\mathrm{Ti}$ 原子的 含量可能影响 $\mathrm{Cu}$ 原子的表面能量, 从而改变了 $\mathrm{Cu}$ 原子的氧化速率，导致当 $\mathrm{TiCuO}$ 薄膜含较多 $\mathrm{Ti}$ 时获 得 $\mathrm{Cu}_{2} \mathrm{O}$, 而 $\mathrm{Ti}$ 含量较少时 $\mathrm{TiCuO}$ 薄膜开始出现 $\mathrm{CuO}$ 。

图 4(b)中, 未掺 $\mathrm{Cu}$ 的 $\mathrm{TiO}_{2}$ 样品中存在 $\mathrm{Ti}^{4+}$ 、 $\mathrm{Ti}^{3+} 、 \mathrm{Ti}^{2+}$ 价态, 峰位分别位于 458.9、457.6、455.2 eV, 对应 $\mathrm{TiO}_{2} 、 \mathrm{Ti}_{2} \mathrm{O}_{3} 、 \mathrm{TiO}$ 结构。当掺入 $\mathrm{Cu}$ 后, 两种 $\mathrm{TiCuO}$ 薄膜中的 $\mathrm{TiO}$ 明显减少, 而 $\mathrm{TiO}_{2}$ 和 $\mathrm{Ti}_{2} \mathrm{O}_{3}$ 增加。文 献[26]报道, 金属 $\mathrm{Cu}$ 的加入可以在一定程度上促进 $\mathrm{Ti}$ 的氧化。在薄膜制备过程中, $\mathrm{Cu}$ 的加入可能促进 了 $\mathrm{Ti}$ 的充分氧化, 故随着 $\mathrm{Cu}$ 含量的增加, $\mathrm{TiCuO}$ 薄 膜中 $\mathrm{TiO}_{2} 、 \mathrm{Ti}_{2} \mathrm{O}_{3}$ 的含量同时增加, 而 $\mathrm{TiO}$ 含量减少。
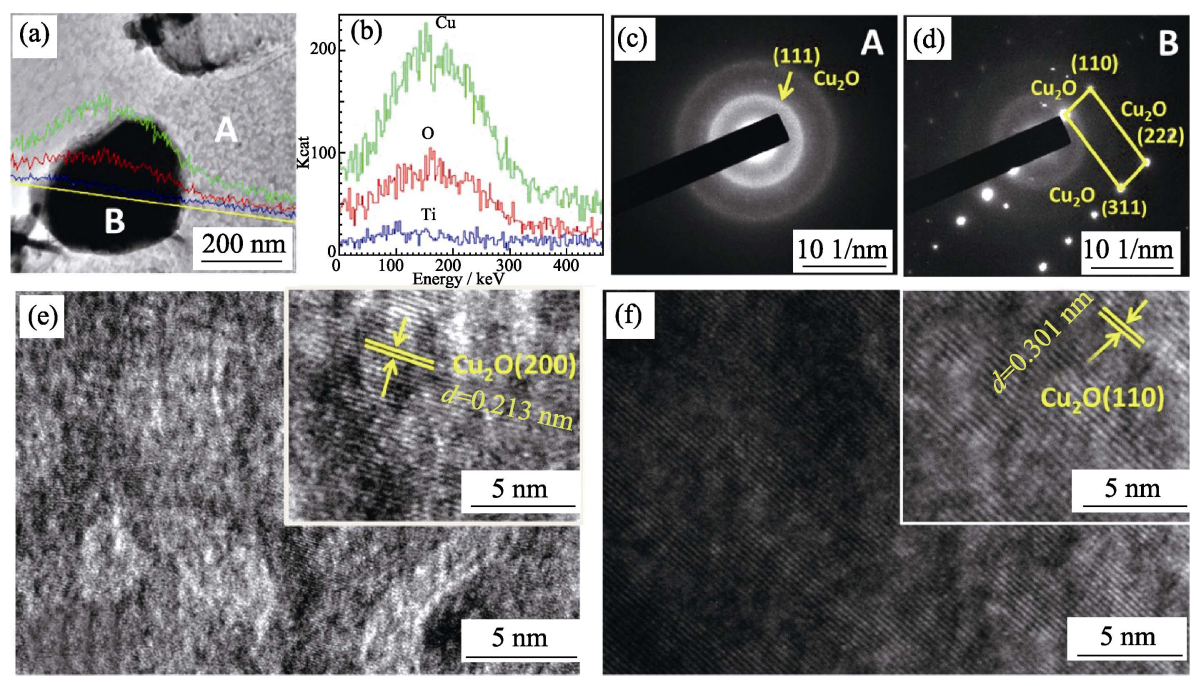

图 $3 \mathrm{TiCuO}-2$ 样品的 TEM 形貌照片(a), $\mathrm{Cu} 、 \mathrm{O} 、 \mathrm{Ti}$ 元素的 EDS 线扫描结果(b), A(c)和 B(d)区域的选区电子衍射图及其 HRTEM 形貌图(e) (f)

Fig. 3 TEM image(a), corresponding line-scanning of $\mathrm{Cu}, \mathrm{O}$, Ti elements (b) across a selected area (yellow line in (a)), the selected area electron diffraction results of A region (c) and B region (d), and their corresponding HRTEM images (e, f) respectively of the TiCuO-2 sample 

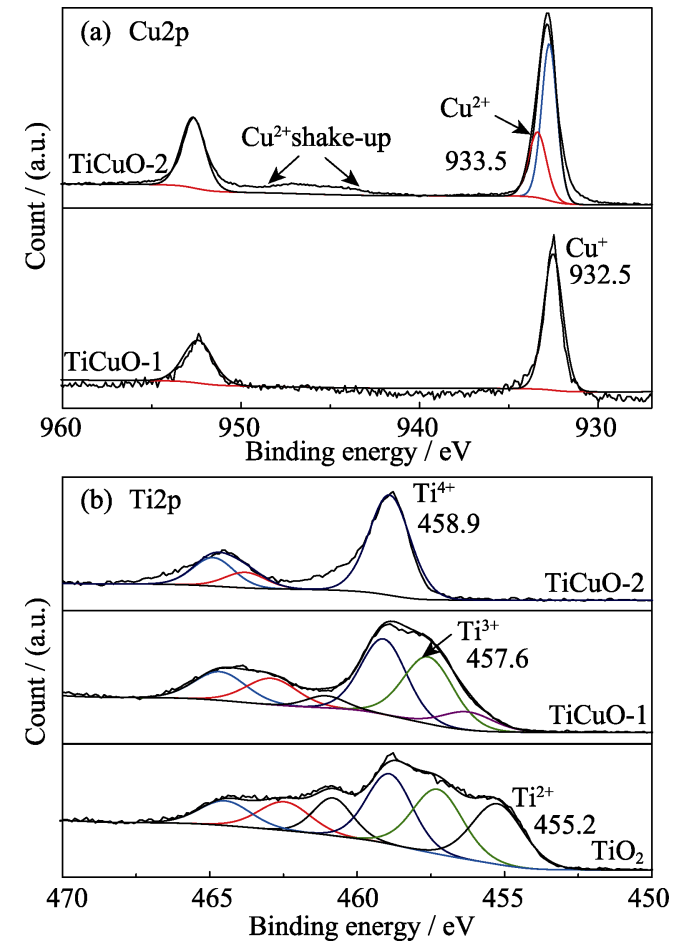

图 4 TiCuO-1 和 $\mathrm{TiCuO}-2$ 的 $\mathrm{Cu}(\mathrm{a})$ 和 $\mathrm{Ti}(\mathrm{b}) \mathrm{XPS}$ 图谱 Fig. $4 \mathrm{Cu} 2 \mathrm{p}$ (a) and Ti2p (b) XPS spectra of TiCuO-1 and TiCuO-2 films

表 $2 \mathrm{TiO}_{2} 、 \mathrm{TiCuO}-1$ 、 $\mathrm{TiCuO}-2$ 样品中的 Ti2p 和 $\mathrm{Cu} 2 \mathrm{p}$ 拟合后的 $\mathbf{T i}^{4+} 、 \mathbf{T i}^{3+} 、 \mathbf{T i}^{2+}$ 在 $\mathbf{T i 2 p}$ 图谱中所占面积的百分含

量及 $\mathrm{Cu}^{2+} 、 \mathrm{Cu}^{+}$在 $\mathrm{Cu} 2 \mathrm{p}$ 图谱中的所占面积的百分含量

Table 2 The area percentages of $\mathrm{Ti}^{4+}, \mathrm{Ti}^{3+}$ and $\mathrm{Ti}^{2+}$ in the Ti2p spectra and the area percentage of $\mathrm{Cu}^{2+}$ and $\mathrm{Cu}^{+}$in Cu2p spectra of $\mathrm{TiO}_{2}$, TiCuO-1 and TiCuO-2 samples

\begin{tabular}{ccccccc}
\hline \multirow{2}{*}{ Sample } & \multicolumn{3}{c}{$\mathrm{Ti} 2 \mathrm{p}$} & & \multicolumn{2}{c}{$\mathrm{Cu} 2 \mathrm{p}$} \\
\cline { 2 - 3 } \cline { 6 - 7 } & $\mathrm{Ti}^{4+}$ & $\mathrm{Ti}^{2+}$ & $\mathrm{Ti}^{3+}$ & & $\mathrm{Cu}^{2+}$ & $\mathrm{Cu}^{+}$ \\
\hline $\mathrm{TiCuO}-2$ & $47 \mathrm{at} \%$ & $53 \mathrm{at} \%$ & 0 & $30.2 \mathrm{at} \%$ & $69.8 \mathrm{at} \%$ \\
$\mathrm{TiCuO}-1$ & $39 \mathrm{at} \%$ & $43 \mathrm{at} \%$ & $18 \mathrm{at} \%$ & $6.2 \mathrm{at} \%$ & $93.8 \mathrm{at} \%$ \\
$\mathrm{TiO}_{2}$ & $32 \mathrm{at} \%$ & $34 \mathrm{at} \%$ & $34 \mathrm{at} \%$ & - & - \\
\hline
\end{tabular}

TEM 结果显示, 含 $\mathrm{Cu}$ 量分别为 $12.4 \mathrm{at} \%$ 和 $23.1 \mathrm{at} \%$ 的 TiCuO-1 和 TiCuO-2 样品均为非晶基体中含有 $\mathrm{Cu}_{2} \mathrm{O}$ 纳米晶粒。XRD 结果也证实了 $\mathrm{TiCuO}$ 薄膜存 在非晶结构(图 1), 但是由于薄膜中的 $\mathrm{Cu}_{2} \mathrm{O}$ 纳米晶 粒很细小且含量少, XRD 中未观察到明显的 $\mathrm{Cu}_{2} \mathrm{O}$ 衍射峰。XPS 结果(图 4, 表 2)也证实了 $\mathrm{Cu}_{2} \mathrm{O}$ 是薄 膜中 $\mathrm{Cu}$ 的主要存在形式。

\section{$2.2 \mathrm{TiCuO}$ 薄膜的腐蚀和 $\mathrm{Cu}$ 离子释放行为}

为了排除薄膜厚度对 $\mathrm{Cu}$ 离子释放结果的影响, 本研究选取了两种 $\mathrm{TiCuO}$ 薄膜的沉积时间, 以获得 相近的薄膜厚度。台阶仪测量结果显示, TiCuO-1 和 $\mathrm{TiCuO}-2$ 薄膜的厚度分别为 $190.3 \mathrm{~nm}$ (沉积时间 $6 \mathrm{~min}$ ) 和 $192.1 \mathrm{~nm}$ (沉积时间 $4 \mathrm{~min}$ )。图 5(A)是 316L SS 和 $316 \mathrm{~L} \mathrm{SS}$ 基体表沉积的 $\mathrm{TiO}_{2} 、 \mathrm{TiCuO}$ 薄膜样品的电
位极化曲线的结果。使用 Tafel 外推法计算的样品自 腐蚀电位和自腐蚀电流密度见表 3。图 5(A)和表 3 结果显示, $\mathrm{TiO}_{2}$ 样品的自腐蚀电流密度比 $316 \mathrm{~L} \mathrm{SS}$ 低，自腐蚀电位比 $316 \mathrm{~L} \mathrm{SS}$ 高。由于材料的自腐蚀 电流密度大小可以反应腐蚀的快慢 ${ }^{[27]}$, 说明 $\mathrm{TiO}_{2}$ 样 品具有较高的抗腐蚀性能。故 $\mathrm{TiO}_{2}$ 薄膜可以作为针 化层和保护层有效地提高 $316 \mathrm{~L} \mathrm{SS}$ 材料的耐腐蚀性 能。表 3 的结果还显示, 两种 $\mathrm{TiCuO}$ 薄膜的自腐蚀 电位较低, 说明 $\mathrm{TiCuO}$ 薄膜与 $316 \mathrm{~L} \mathrm{SS}$ 和 $\mathrm{TiO}_{2}$ 薄膜 相比较, 更易发生腐蚀。且 $\mathrm{TiCuO}-1$ 和 $\mathrm{TiCuO}-2$ 样 品的自腐蚀电流密度分别为 0.62 和 $3.89 \mu \mathrm{A} / \mathrm{cm}^{2}$, 均 高于 $316 \mathrm{~L} \mathrm{SS}$ 样品的自腐蚀的电流密度 $0.49 \mu \mathrm{A} / \mathrm{cm}^{2}$ 。含 $\mathrm{Cu} 12.4 \mathrm{at} \%$ 的 $\mathrm{TiCuO}-1$ 样品在 $0.43 \mathrm{~V}$ 时产生了点蚀 现象, 含 $\mathrm{Cu}$ 更多的 $\mathrm{TiCuO}-2$ 样品在更低的腐蚀电压 $(0.05 \mathrm{~V})$ 下也出现了点蚀现象, 并且自腐蚀电流密 度突增后减少，提示薄膜发生了腐蚀现象和薄膜的 自修复效应 ${ }^{[28]}$ 。以上结果说明 $\mathrm{TiCuO}$ 样品的耐腐蚀 性能高于 $316 \mathrm{~L} \mathrm{SS}$, 并且含 $\mathrm{Cu}$ 量较多的 $\mathrm{TiCuO}-2$ 样品 更易被腐蚀。采用 SEM 和 EDS 对电化学腐蚀后 TiCuO-2 样品的表面形貌和成分分别进行了分析。
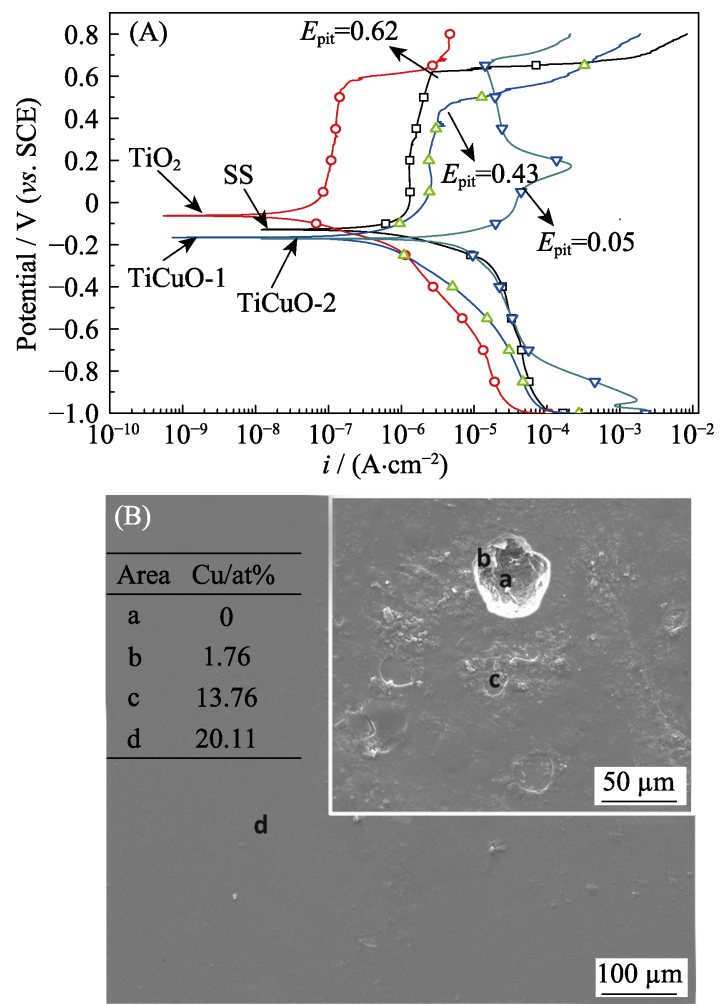

图 $5 \mathrm{TiO}_{2} 、 \mathrm{TiCuO}$ 样品和 $\mathrm{SS}$ 的电化学腐蚀极化曲线(A)及 $\mathrm{TiCuO}-2$ 样品的腐蚀形貌图和 $\mathrm{a}, \mathrm{b}, \mathrm{c}, \mathrm{d}$ 四个不同腐蚀区域的 EDS 结果(B)

Fig. 5 Electrochemical corrosion polarization curves of $\mathrm{TiO}_{2}$, $\mathrm{TiCuO}$ and $316 \mathrm{~L}$ SS samples (A) and morphology of the TiCuO-2 sample with its EDS results of the four different corroded areas labeled with a, b, c and d (B) 
表 $3 \mathrm{TiO}_{2} 、 \mathrm{TiCuO}$ 和 SS 的自腐蚀电位 $\left(E_{\mathrm{corr}}\right)$ 及其自腐蚀电流密度 $\left(I_{\text {corr }}\right)$

Table 3 Corrosion potential $\left(E_{\text {corr }}\right)$ and current densities ( $\left.I_{\text {corr }}\right)$ of SS, $\mathrm{TiO}_{2}$ and $\mathrm{TiCuO}$ films derived from potentiodynamic polarization curves

\begin{tabular}{ccccc}
\hline Sample & $\mathrm{SS}$ & $\mathrm{TiO}_{2}$ & $\mathrm{TiCuO}-1$ & $\mathrm{TiCuO}-2$ \\
\hline$E_{\text {corr }} / \mathrm{V}(v s . \mathrm{SCE})$ & -0.13 & -0.06 & -0.16 & -0.17 \\
$I_{\text {corr }} /\left(\mu \mathrm{A} \cdot \mathrm{cm}^{-2}\right)$ & 0.49 & 0.03 & 0.62 & 3.89 \\
\hline
\end{tabular}

结果显示, 薄膜中大部分位置较为平整, 少数地方 出现了腐蚀坑(图 5(B))。材料表面腐蚀较为严重的 区域 $(a 、 b 、 c)$, 其 $\mathrm{Cu}$ 含量明显下降; 而腐蚀较轻区 域(d)的区域, 其 $\mathrm{Cu}$ 的含量较大并接近于未腐蚀的 样品含量。由此推测, 薄膜主要从富 $\mathrm{Cu}$ 区发生腐蚀 并释放出 $\mathrm{Cu}$ 离子。由 TEM 和 XPS 分析结果可知, $\mathrm{TiCuO}$ 样品中主要存在 $\mathrm{Cu}_{2} \mathrm{O}$ 结构, 也存在氧化钛。 由于铜氧化物比氧化钛更容易被腐蚀 ${ }^{[29]}$, 因此薄 膜中的富 $\mathrm{Cu}$ 区可能是薄膜开始发生腐蚀的主要区 域。含 $\mathrm{Cu} 23.1 \mathrm{at} \%$ 的 TiCuO-2 薄膜中含较多的富 $\mathrm{Cu}$ 区，而氧化钛钝化层含量相对更少，故更易发生 腐蚀。

图 6(a)为采用 ICP-MS 方法, 检测了在标准磷酸 缓冲盐溶液 $(\mathrm{pH}=7.4)$ 中分别浸泡 $30 \mathrm{~min}$ 和 $1 、 3 、 5 、$ $15 、 30 \mathrm{~d}$ 后 $\mathrm{TiCuO}$ 薄膜累积释放的 $\mathrm{Cu}$ 离子浓度。 结果显示, 随着薄膜浸泡时间的延长, $\mathrm{Cu}$ 离子累积 释放浓度逐渐增加。从 $\mathrm{TiCuO}$ 薄膜的 $\mathrm{Cu}$ 离子平均 释放速率(图 6(b)) 可以看出当 $\mathrm{TiCuO}-1$ 和 $\mathrm{TiCuO}-2$ 样品开始浸泡 $30 \mathrm{~min}$ 时, 薄膜中 $\mathrm{Cu}$ 离子的释放速 率均最快, 分别为 $1.66 \times 10^{-4} \mu \mathrm{mol} /\left(\mathrm{cm}^{2} \cdot \mathrm{h}\right)$ 和 $1.88 \times$ $10^{-4} \mu \mathrm{mol} /\left(\mathrm{cm}^{2} \cdot h\right)$ 。这提示 $\mathrm{TiCuO}$ 薄膜浸入标准磷酸 缓冲盐溶液后, 在开始阶段发生了 $\mathrm{Cu}$ 离子的爆发 性释放。在较多含 $\mathrm{Cu}$ 材料中均具有这种懪发性释 放现象 ${ }^{[26,28,30]}$ 。随着浸泡时间延长, $\mathrm{TiCuO}-1$ 和 $\mathrm{TiCuO}-2$ 样品中 $\mathrm{Cu}$ 离子平均释放速率都逐渐降低, 随后 $\mathrm{TiCuO}$ 薄膜均表现出 $30 \mathrm{~d}$ 稳定的 $\mathrm{Cu}$ 离子释放 速率。在浸泡时间长达 $30 \mathrm{~d}$ 时, TiCuO-1 和 TiCuO-2 样品累积释放的 $\mathrm{Cu}$ 离子浓度分别约为 $1.5 \mu \mathrm{mol} / \mathrm{L}$ 和 $5 \mu \mathrm{mol} / \mathrm{L}$, 含 $\mathrm{Cu}$ 更多的 TiCuO-2 样品累积释放 $\mathrm{Cu}$ 离子浓度是 TiCuO-1 样品的 3.4 倍, 表明含 $\mathrm{Cu}$ 更多的 $\mathrm{TiCuO}$ 薄膜释放出了更多的 $\mathrm{Cu}$ 离子。薄膜 的电化学腐蚀结果显示(图 5(a)), 含 $\mathrm{Cu}$ 更多的 $\mathrm{TiCuO}$ 样品更容易被腐蚀, 这说明 $\mathrm{TiCuO}$ 薄膜的 $\mathrm{Cu}$ 离子释放与其腐蚀行为密切相关。文献[28]显示: $\mathrm{Cu}$ 离子释放速率受到薄膜的厚度、薄膜的晶体结构和 $\mathrm{Cu}$ 含量浓度等影响。含 $\mathrm{Cu}$ 量为 $12.4 \mathrm{at} \%$ 的 $\mathrm{TiCuO}-1$ 和 23.1 at \% 的 TiCuO-2 样品厚度相似 $(200 \mathrm{~nm})$, 结 构一致(非晶基体中含有少量的纳米 $\mathrm{Cu}_{2} \mathrm{O}$ 晶粒)。较
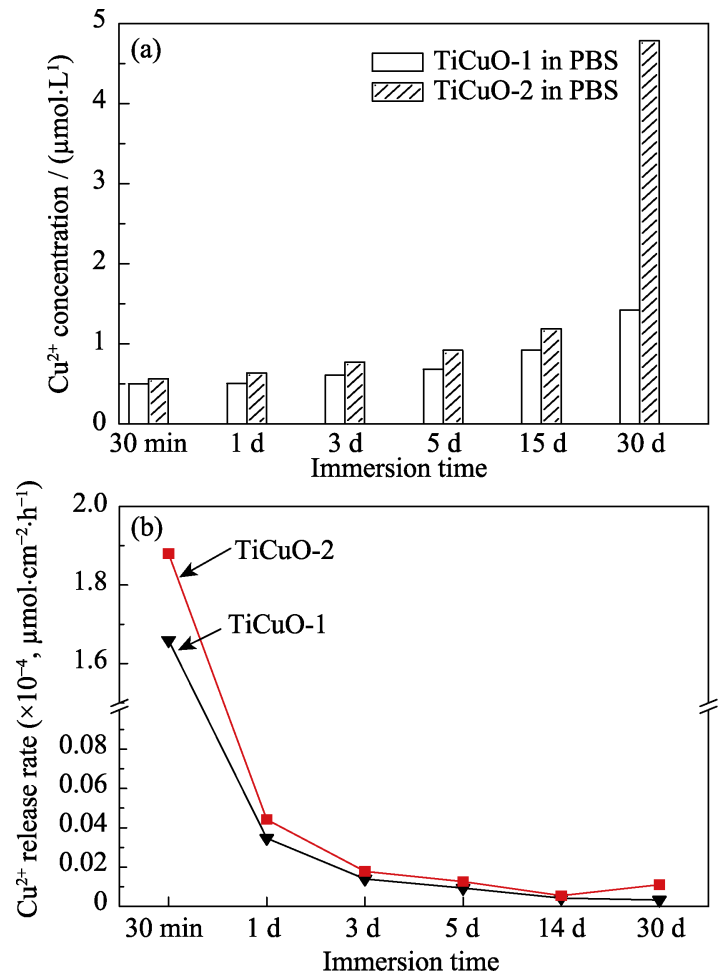

图 6 TiCuO-1 和 $\mathrm{TiCuO}-2$ 样品释放 $\mathrm{Cu}$ 离子累积浓度(a)和 $\mathrm{Cu}$ 离子平均释放速率(b)

Fig. 6 Accumulated $\mathrm{Cu}$ ions concentration (a) and release rate (b) of $\mathrm{Cu}$ ions released from TiCuO- 1 and $\mathrm{TiCuO}-2$ samples

高的 $\mathrm{Cu}$ 含量和存在铜氧化物的富 $\mathrm{Cu}$ 区加剧了薄膜 的腐蚀行为, 从而提高了 $\mathrm{Cu}$ 离子释放速率。

\section{$2.3 \mathrm{TiCuO}$ 薄膜表面内皮细胞相容性分析}

图 7(a) 是在 $\mathrm{TiO}_{2}$ 和 $\mathrm{TiCuO}$ 样品表面体外静态培 养内皮细胞 1 和 $3 \mathrm{~d}$ 后, CCK-8 试剂盒检测的内皮细 胞活性结果。含 $\mathrm{Cu} 12.4 \mathrm{at} \%$ 的 $\mathrm{TiCuO}-1$ 样品在培养 内皮细胞 $3 \mathrm{~d}$ 后, 其细胞活性比 $\mathrm{TiO}_{2}$ 样品有显著增 加。所有样品表面的内皮细胞形态均呈现正常的椭圆 形, 有正常内皮细胞典型的“铺路石”生长形态(图 7(b))。 根据 ICP-MS 结果(图 6(a)), TiCuO-1 和 TiCuO-2 样 品在模拟体液中浸泡 1 至 $30 \mathrm{~d}$ 时, 它们释放的 $\mathrm{Cu}$ 离 子的累积浓度范围分别约 $0.5 \sim 1.5 \mu \mathrm{mol} / \mathrm{L}$ 和 $0.7 \sim$ $5 \mu \mathrm{mol} / \mathrm{L}$ 。文献报道 $\mathrm{Cu}^{2+}$ 和 $\mathrm{Cu}^{+}$离子均能促进内皮 细胞增殖 ${ }^{[31]}$, 并且在微摩尔浓度范围内的 $\mathrm{Cu}$ 离子可 以促进主动脉内皮细胞的增殖和迁移 ${ }^{[9,31-32]}$ 。

为了进一步分析 $\mathrm{Cu}$ 离子对内皮细胞的影响, 采用 CCK-8 试剂盒检测了含不同 $\mathrm{CuCl}_{2}$ 浓度培养液 培养的内皮细胞活性，并对其进行了苂光染色。 图 8(a) (b) 是不同浓度 $\mathrm{CuCl}_{2}$ 条件下培养内皮细胞 1 和 $3 \mathrm{~d}$ 后细胞活性结果, 及 $3 \mathrm{~d}$ 时细胞的苂光显微镜 下的形态。结果表明: 1 15 $\mu \mathrm{mol} / \mathrm{L}$ 的 $\mathrm{CuCl}_{2}$ 溶液显 著促进内皮细胞活性, 当浓度大于 $50 \mu \mathrm{mol} / \mathrm{L}$ 时没有 明显促进作用，浓度高于 $500 \mu \mathrm{mol} / \mathrm{L}$ 时明显抑 

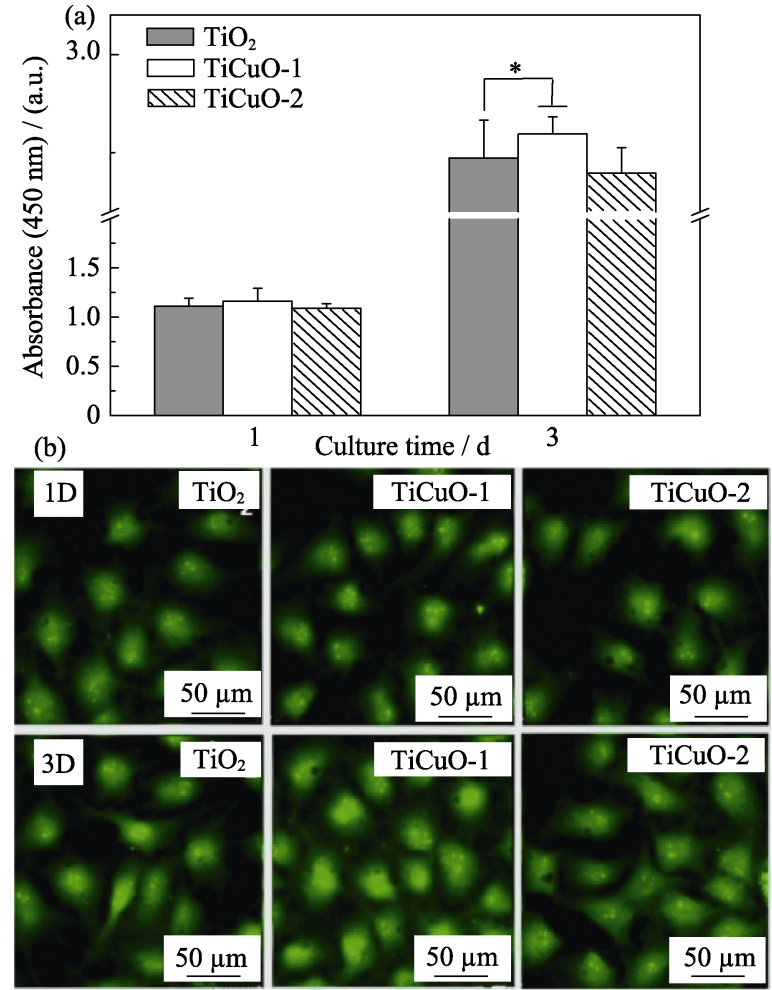

图 $7 \mathrm{TiO}_{2} 、 \mathrm{TiCuO}$ 样品体外静态培养内皮细胞的细胞活性 (a)和罗丹明苂光染色照片 (b)

Fig. 7 ECs viability detected by CCK-8 assay (a) and fluorescence microscopic images stained by rhodamine (b) after being cultured on the samples of $\mathrm{TiO}_{2}$ and $\mathrm{TiCuO}$
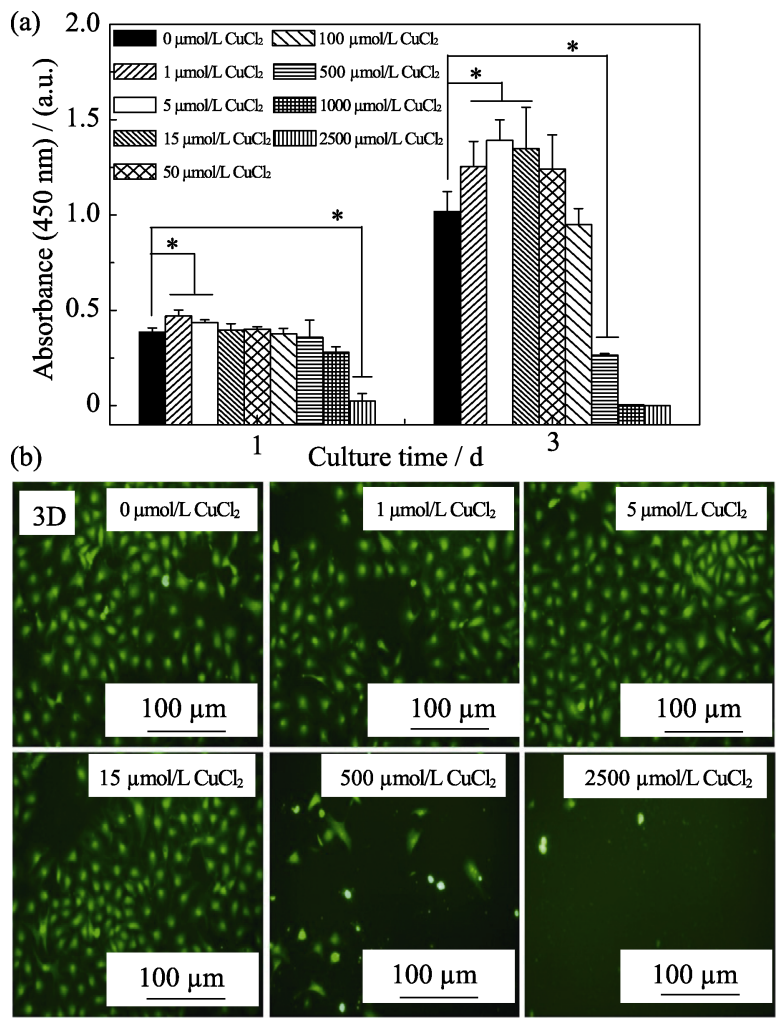

图 $8 \quad \mathrm{TiO}_{2} 、 \mathrm{TiCuO}$ 样品体外静态培养内皮细胞的细胞活性(a) 和罗丹明荧光染色照片(b)

Fig. 8 ECs viability detected by CCK-8 assay (a) and fluorescence microscopic images stained by rhodamine (b) after being cultured under condition medium with $\mathrm{CuCl}_{2}$ solution at different concentration
制了内皮细胞活性，说明 $\mathrm{CuCl}_{2}$ 浓度明显影响了内 皮细胞活性。 $\mathrm{TiCuO}$ 薄膜表面培养 $3 \mathrm{~d}$ 后的内皮细 胞结果(图 7)显示: 与 $\mathrm{TiO}_{2}$ 样品相比较, $\mathrm{TiCuO}-1$ $(12.4 \mathrm{at} \% \mathrm{Cu})$ 的样品表面的内皮细胞活性显著性提 高, 而含 $\mathrm{Cu}$ 量较高 $(23.1 \mathrm{at} \% \mathrm{Cu}$ ) 的 TiCuO-2 样品表 面没有显著性增加。这是因为 $\mathrm{TiCuO}-1$ 薄膜表面释 放出一定浓度的 $\mathrm{Cu}$ 离子, 提高了内皮细胞活性。而 含 $\mathrm{Cu}$ 量更高的 $\mathrm{TiCuO}-2$ 样品更易被腐蚀并释放出 较高浓度的 $\mathrm{Cu}$ 离子, 没有显著提高其内皮细胞活 性。文献[33]报道, 浸入细胞培养液 DMEM 中的纳 米 $\mathrm{Cu}$ 和 $\mathrm{CuO}$ 颗粒所释放的 $\mathrm{Cu}$ 离子量比浸入在 PBS 中显著增加。因此, $\mathrm{TiCuO}$ 薄膜在细胞培养液中释放 出的 $\mathrm{Cu}$ 离子浓度可能与在 PBS 中存在一定差异。 但在薄膜表面与 $\mathrm{CuCl}_{2}$ 溶液中分别培养内皮细胞的 结果, 均发现 $\mathrm{Cu}$ 离子浓度在一定范围时可以促进 内皮细胞的活性, 而浓度较高时, 则显示出抑制作 用。这些结果提示, $\mathrm{TiCuO}$ 薄膜释放出的 $\mathrm{Cu}$ 离子浓 度影响了内皮细胞活性。另外, $\mathrm{TiCuO}-2 、 \mathrm{TiCuO}-1$ 和 $\mathrm{TiO}_{2}$ 薄膜表面的水接触角分别约为 $(98 \pm 0 .)^{\circ}$ 、 $(93 \pm 0.1)^{\circ} 、(91 \pm 0.2)^{\circ}$ 。材料表面的润湿性能会影响 内皮细胞行为 ${ }^{[34-35]}$, 具有一定亲疏水平衡的表面最 有利于细胞生长 ${ }^{[36-37]}$ 。除了释放更多的 $\mathrm{Cu}$ 离子外, TiCuO-2 较为疏水的表面也可能导致了内皮细胞活 性没有显著性的增加。而 $\mathrm{TiCuO}-1$ 薄膜样品表面的 润湿性等同于 $\mathrm{TiO}_{2}$ 薄膜表面, 释放出适当浓度的 $\mathrm{Cu}$ 离子促进和改善了内皮细胞活性。

\section{3 结论}

采用直流磁控溅射技术成功地在 $\mathrm{Si}$ 片和 $316 \mathrm{~L}$ $\mathrm{SS}$ 基体上沉积出 $\mathrm{TiCuO}$ 薄膜。 $\mathrm{TiCuO}$ 薄膜为非晶 基体, 其中含有 $\mathrm{Cu}_{2} \mathrm{O}$ 纳米晶粒。 $\mathrm{TiCuO}$ 薄膜中富 $\mathrm{Cu}$ 区引起了薄膜腐蚀, $\mathrm{Cu}$ 含量较高的 $\mathrm{TiCuO}$ 薄膜 较易被腐蚀, 并能释放出较多 $\mathrm{Cu}$ 离子。含 $\mathrm{Cu}$ 量为 $12.4 \mathrm{at} \%$ 的 $\mathrm{TiCuO}$ 薄膜显著提高了内皮细胞体外培 养状态下的细胞活性, 薄膜释放出一定浓度的 $\mathrm{Cu}$ 离子可以促进内皮细胞活性。 $\mathrm{TiCuO}$ 薄膜的含 $\mathrm{Cu}$ 量和显微结构影响了 $\mathrm{Cu}$ 离子释放, 并影响了内皮 细胞活性。

\section{参考文献:}

[1] WARD M R, STEWART D J, KUTRYK M J. Endothelial progenitor cell therapy for the treatment of coronary disease, acute MI, and pulmonary arterial hypertension: current perspectives. Catheterization and Cardiovascular Interventions, 2007, 70(7): 983-998.

[2] NISIO D M, MIDDELDORP S, BÜLLER H R. Direct thrombin 
inhibitors. New England Journal of Medicine, 2005, 353(10): 1028-1040.

[3] AKAHORI T, NIINOMI M. Fracture characteristics of fatigued Ti-6Al-4V ELI as an implant material. Materials Science \& Engineering A, 1998, 243(1/2): 237-243.

[4] LENG Y X, CHEN J Y, YANG P, et al. Structure and properties of passivating titanium oxide films fabricated by DC plasma oxidation. Surface \& Coatings Technology, 2003, 166(2/3): 176-182.

[5] TSYGANOV I A, MAITZ M F, RICHTER E, et al. Hemocompatibility of titanium-based coatings prepared by metal plasma immersion ion implantation and deposition. Nuclear Instruments \& Methods in Physics Research, 2007, 257(1/2): 122-127.

[6] KLESZCZEWSKI T, MODZELEWSKA B, BAL W, et al. Cu(II) complexation does not affect oxytocin action on pregnant human myometrium in vitro. Reproductive Toxicology, 2015, 59: 60-65.

[7] AGGETT P J. An overview of the metabolism of copper. European Journal of Medical Research, 1999, 4(6): 214-216.

[8] GAETKE L M, CHOW C K. Copper toxicity, oxidative stress, and antioxidant nutrients. Toxicology, 2003, 189(1/2): 147-163.

[9] HU G. Copper stimulates proliferation of human endothelial cells under culture. Journal of Cellular Biochemistry, 1998, 69(3): 326335.

[10] REN L, XU L, FENG J, et al. In vitro study of role of trace amount of $\mathrm{Cu}$ release from $\mathrm{Cu}$-bearing stainless steel targeting for reduction of in-stent restenosis. Journal of Materials Science Materials in Medicine, 2012, 23(5): 1235-1245.

[11] SEN C K, KHANNA S, VENOJARVI M, et al. Copper-induced vascular endothelial growth factor expression and wound healing. Am. J. Physiol.-Heart Circ. Physiol., 2002, 282(5): H1821-H1827.

[12] YIN R, LING L, XIANG Y, et al. Enhanced photocatalytic reduction of chromium (VI) by $\mathrm{Cu}$-doped $\mathrm{TiO}_{2}$ under UV-A irradiation. Separation \& Purification Technology, 2017, 190: 53-59.

[13] ZONG M, LONG B, LIU Y, et al. Antibacterial ability and angiogenic activity of $\mathrm{Cu}-\mathrm{TiO}_{2}$ nanotube arrays. Materials Science \& Engineering C, 2017, 71: 93-99.

[14] NORAMBUENA G A, PATEL R, KARAU M, et al. Antibacterial and bio compatible titanium-copper oxide coating may be a potential strategy to reduce periprosthetic infection: an in vitro study. Clinical Orthopaedics \& Related Research, 2016, 475(3): 1-11.

[15] WANG H, LI Y, BA X, et al. $\mathrm{TiO}_{2}$ thin films with rutile phase prepared by DC magnetron co-sputtering at room temperature: effect of Cu incorporation. Applied Surface Science, 2015, 345: 49-56.

[16] TOMOLYA K, JANOVSZKY D, SYCHEVA A, et al. Peculiarities of ball-milling induced crystalline-amorphous transformation in Cu-Zr-Al-Ni-Ti alloys. Intermetallic, 2015, 65: 117-121.

[17] NABIAŁEK M. Soft magnetic and microstructural investigation in Fe-based amorphous alloy. Journal of Alloys \& Compounds, 2015, 642: 98-103.

[18] OLEKSAK RP, DEVARAJ A, HERMAN GS. Atomic-scale structural evolution of Ta-Ni-Si amorphous metal thin films. Materials Letters, 2016, 164: 9-14.

[19] TSENG I H, WU J C S, CHOU H Y. Effects of Sol-Gel procedures on the photocatalysis of $\mathrm{Cu} / \mathrm{TiO}_{2}$ in $\mathrm{CO}_{2}$ photoreduction. Journal of Catalysis, 2004, 221(2): 432-440.

[20] SUN C, ZHU J, LV Y, et al. Dispersion, reduction and catalytic performance of $\mathrm{CuO}$ supported on $\mathrm{ZrO}_{2}$-doped $\mathrm{TiO}_{2}$ for $\mathrm{NO}$ removal by CO. Applied Catalysis B: Environmental, 2011, 103(1): 206-220.

[21] LIU J, LI X, ZHAO Q, et al. The selective catalytic reduction of $\mathrm{NO}$ with propene over $\mathrm{Cu}$-supported Ti-Ce mixed oxide catalysts: promotional effect of ceria. Journal of Molecular Catalysis A: Chemical, 2013, 378: 115-123.

[22] LONG R, ENGLISH N J. Electronic properties of anatase- $\mathrm{TiO}_{2}$, co-doped by cation-pairs from hybrid density functional theory calculations. Chemical Physics Letters, 2011, 513(4): 218-223.

[23] NAKAYAMA S, KIMURA A, SHIBATA M, et al. Voltammetric characterization of oxide films formed on copper in air. Journal of the Electrochemical Society, 2001, 148(11): B467-B472.

[24] NAKAYAMA S, NOTOYA T, OSAKAI T. Highly selective determination of copper corrosion products by voltammetric reduction in a strongly alkaline electrolyte. Analytical Sciences the International Journal of the Japan Society for Analytical Chemistry, 2012, 28(4): 323.

[25] BELOUSOV V V. Mechanisms of accelerated oxidation of copper in the presence of molten oxides. Oxidation of Metals, 2007, 67(5/6): 235-250.

[26] AL-MAYOUF A M, AL-SWAYIH A A, AL-MOBARAK N A, et al. Corrosion behavior of a new titanium alloy for dental implant applications in fluoride media. Materials Chemistry and Physics, 2004, 86(2/3): 320-329.

[27] LIU H, LENG Y, HUANG N. Corrosion resistance of Ti-O film modified $316 \mathrm{~L}$ stainless steel coronary stents in vitro. Journal of Materials Engineering \& Performance, 2012, 21(3): 424-428.

[28] LIU H, ZHANG D, FENG S, et al. Corrosion and ion release behavior of $\mathrm{Cu} / \mathrm{Ti}$ film prepared via physical vapor deposition in vitro as potential biomaterials for cardiovascular devices. Applied Surface Science, 2012, 258(19): 7286-7291.

[29] JACEK BANAS, ANDRZEJ MAZURKIEWICZ. The effect of copper on passivity and corrosion behaviour of ferritic and ferriticaustenitic stainless steels. Materials Science and Engineering: A, 2000, 277(1/2): 183-191.

[30] STRANAK V, WULFF H, REBL H, et al. Deposition of thin titanium copper films with antimicrobial effect by advanced magnetron sputtering methods. Materials Science \& Engineering C, 2011, 31(7): 1512-1519.

[31] SEO Y, CHO Y S, HUH Y D, et al. Copper ion from $\mathrm{Cu}_{2} \mathrm{O}$ crystal induces AMPK-mediated autophagy via superoxide in endothelial cells. Molecules \& Cells, 2016, 39(3): 195-203.

[32] LIU H, PAN C, ZHOU S, et al. Improving hemocompatibility and accelerating endothelialization of vascular stents by a copper-titanium film. Materials Science \& Engineering C Materials for Biological Applications, 2016, 69: 1175-1182.

[33] MIDANDER K, CRONHOLM P, KARLSSON H L, et al. Surface characteristics, copper release, and toxicity of nano-and micrometersized copper and copper(II) oxide particles: a cross-disciplinary study. Small, 2009, 5(3): 389-399.

[34] ARIMA Y, IWATA H. Effect of wettability and surface functional groups on protein adsorption and cell adhesion using well-defined mixed self-assembled monolayers. Biomaterials, 2007, 28(20): 3074-3082.

[35] ESHAGHI A, ESHAGHI A. Preparation and hydrophilicity of $\mathrm{TiO}_{2}$ Sol-Gel derived nanocomposite films modified with copper loaded $\mathrm{TiO}_{2}$ nanoparticles. Materials Research Bulletin, 2011, 46(12): $2342-2345$.

[36] HUANG L, NING C Q, DING D Y, et al. Wettability and in vitro bioactivity of doped $\mathrm{TiO}_{2}$ nanotubes. Journal of Inorganic Materials, 2010, 25(7): 775-779.

[37] BRAMMER K S, OH S, GALLAGHER J O, et al. Enhanced cellular mobility guided by $\mathrm{TiO}_{2}$ nanotube surfaces. Nano Letters, 2008, 8(3): 786-793. 